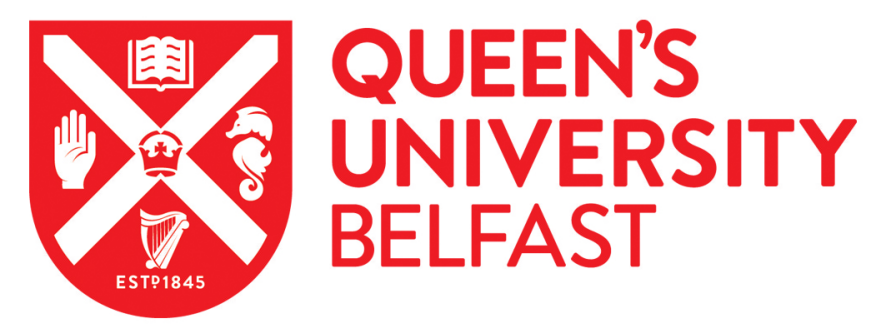

\title{
The Role of Ultraviolet Photons in Circumstellar Astrochemistry
}

Millar, T. J. (2020). The Role of Ultraviolet Photons in Circumstellar Astrochemistry. Chinese Journal of Chemical Physics, 33, 668-679.

Published in:

Chinese Journal of Chemical Physics

Document Version:

Peer reviewed version

Queen's University Belfast - Research Portal:

Link to publication record in Queen's University Belfast Research Portal

Publisher rights

OCopyright 2020 Chinese Physical Society

This work is made available online in accordance with the publisher's policies. Please refer to any applicable terms of use of the publisher.

\section{General rights}

Copyright for the publications made accessible via the Queen's University Belfast Research Portal is retained by the author(s) and / or other copyright owners and it is a condition of accessing these publications that users recognise and abide by the legal requirements associated with these rights.

Take down policy

The Research Portal is Queen's institutional repository that provides access to Queen's research output. Every effort has been made to ensure that content in the Research Portal does not infringe any person's rights, or applicable UK laws. If you discover content in the Research Portal that you believe breaches copyright or violates any law, please contact openaccess@qub.ac.uk. 


\section{The Role of Ultraviolet Photons in Circumstellar Astrochemistry}

T J Millar ${ }^{1, a)}$

Astrophysics Research Centre, School of Mathematics and Physics, Queen's University Belfast, Belfast BTr7 1NN, UK

(Dated: 14 October 2020)

Stars with masses between 1 and 8 solar masses $\left(\mathrm{M}_{\odot}\right)$ lose large amounts of material in the form of gas and dust in the late stages of stellar evolution, during their Asymptotic Giant Branch phase. Such stars supply up to $35 \%$ of the dust in the interstellar medium and thus contribute to the material out of which our solar system formed. In addition, the circumstellar envelopes of these stars are sites of complex, organic chemistry with over 80 molecules detected in them. We show that internal ultraviolet photons, either emitted by the star itself or from a close-in, orbiting companion, can significantly alter the chemistry that occurs in the envelopes particularly if the envelope is clumpy in nature. At least for the cases explored here, we find that the presence of a stellar companion, such as a white dwarf star, the high flux of UV photons destroys $\mathrm{H}_{2} \mathrm{O}$ in the inner regions of carbon-rich AGB stars to levels below those observed and produces species such as $\mathrm{C}^{+}$deep in the envelope in contrast to the expectations of traditional descriptions of circumstellar chemistry.

a)Tom.Millar@qub.ac.uk 


\section{INTRODUCTION}

Mass loss from stars on the Asymptotic Giant Branch (AGB) phase typically lasts a few times $10^{5}$ years. Despite this short astronomical time-scale, this period of stellar evolution is one of intense study for a number of reasons: (i) their atmospheres are a major site of dust formation in the Universe; (ii) through their stellar winds, they provide at least $30 \%$ of the dust found in the interstellar medium; (iii) the measurement of isotopic ratios from molecular lines allows one to probe nucleosynthetic processes deep inside the star; (iv) a simple dynamical environment that allows physical properties of the wind, such as density, temperature, expansion velocities and chemical abundances, to be determined as a function of radial distance through the circumstellar envelope (CSE), and (v) a relatively simple 'astrochemical laboratory' in the outer CSE, having spherical symmetry, a large-scale constant-velocity expansion and with well-defined chemical processes that are dependent on radial distance from the star.

These CSEs, particularly those around carbon-rich AGB stars - notably IRC+10216 (also known as CW Leo) - are rich in linear carbon-chain species, such as $\mathrm{C}_{n} \mathrm{H}(n=1-8), \mathrm{C}_{n} \mathrm{O}(n=$ $1-3,5), \mathrm{C}_{n} \mathrm{~N}(n=1-3,5), \mathrm{C}_{n} \mathrm{~S}(n=1-3,5), \mathrm{HC}_{n} \mathrm{~N}(n=1-5,7,9)$, the anions $\mathrm{C}_{n} \mathrm{~N}^{-}(n=1,3,5)$ and $\mathrm{C}_{n} \mathrm{H}^{-}(n=4,6,8)$. One should note, however, that the linear nature of these species provides a favourable aspect for radio astronomical detection of rotational transitions since it minimises the value of the rotational partition function - the intrinsic energy of rotation is spread over fewer energy levels than is the case for non-linear molecules.

The CSE around IRC+10216 is a particular object of interest since over 80 molecules have been detected in it, indeed many for the very first, and some the only, time. In part, this is due to the carbon-rich nature of the wind since the carbon atom bonds easily to itself and other elements, in part to its very high mass-loss rate, $\dot{M}$, in excess of $10^{-5}$ solar masses

per year $\left(\mathrm{M}_{\odot} \mathrm{yr}^{-1}\right)$, and in part to its proximity to Earth, around 130 parsecs (pc). It has a huge CO envelope extending to an angular radius of some 200 arcsec, or about 1 light year. At its distance from Earth, 1 arcsec is equivalent to about $2 \times 10^{15} \mathrm{~cm}$. Given its expansion velocity, $v_{e}$ of about $15 \mathrm{~km} \mathrm{~s}^{-1}$, material takes about $10^{4} \mathrm{yr}$ to move from the dust formation zone at a few stellar radii $\left(\mathrm{R}_{*}\right)$ to the interstellar medium.

Early observations of this source indicated that molecules could be grouped into two categories, one in which they appeared close to the stellar photosphere and the other in 
which they appeared in shells of radii $12-20 \operatorname{arcsec}$ and with widths of 2-4 $\operatorname{arcsec}{ }^{1-4}$. These observations were shown to be consistent with a model in which parent species, such as $\mathrm{C}_{2} \mathrm{H}_{2}, \mathrm{HCN}$ and $\mathrm{CO}$, were formed in the high-temperature chemistry near the photosphere, accelerated to terminal velocity through collisions with dust grains, and photodissociated by interstellar UV photons as they moved outwards into a region of high interstellar UV flux. The formation of radical daughter products leads to a rapid chemistry that builds up more complex molecules before they too are photodissociated by the interstellar field as they move outward through the envelope. The high mass-loss rate of IRC +10216 ensures that densities are high and reactive time-scales short, on the order of a few hundred years, in this interaction zone.

The early models describing molecule formation in the outer CSE were all based on this description $^{5-11}$. The detection of abundant hot water at a temperature of several hundred degrees, with a fractional abundance of around $10^{-7}$, in IRC $+10216^{12}$ led to a fundamental reappraisal of these models since they predicted most oxygen would be locked up in CO and very little would be free to form water in the warm inner CSE. Although a number of suggestions were made for the formation of this hot water, that currently favoured is that the CSE is clumpy enough to allow interstellar UV photons to penetrate unhindered to the dense inner envelope. Once in this inner region, the photons can dissociate ${ }^{13} \mathrm{CO}$ and $\mathrm{SiO}$ with the released $\mathrm{O}$ atoms able to react with $\mathrm{H}_{2}$ to form water ${ }^{13}$. The importance of non-Local Thermodynamic Equilibrium (LTE) chemistry in the inner CSE was strengthened further by the detection ${ }^{14}$ of methyl cyanide, $\mathrm{CH}_{3} \mathrm{CN}$, on an angular scale of 1-2 arcsec, a molecule which is not made efficiently through LTE chemistry.

\section{CHEMICAL STRUCTURE}

In the following sub-sections, I describe briefly the dominant chemistry as a function of radial distance in the CSE.

\section{A. The Photosphere}

The photospheres of AGB stars consists of hot $(\sim 2000-3000 \mathrm{~K})$, dense $\left(n=10^{12}-10^{14}\right.$ $\mathrm{cm}^{-3}$ ) gas in which LTE determines the chemical composition of the gas. After $\mathrm{H}_{2}$, $\mathrm{CO}$ is 
the most stable molecule and essentially takes up all of the available oxygen in an C-rich star and all the available carbon in an O-rich star. The excess abundance of oxygen in the latter results in large abundances of molecules such as $\mathrm{H}_{2} \mathrm{O}, \mathrm{SiO}$ and $\mathrm{SO}$, while the excess of carbon in the former leads to high abundances of $\mathrm{C}_{2} \mathrm{H}_{2}$ and $\mathrm{HCN}$.

Sharp and Wasserburg ${ }^{15}$ present the results of LTE abundances and condensation sequences of molecules as a function of the $\mathrm{C} / \mathrm{O}$ ratio. Although these condensation calculations can give some information on the refractory species in which the elements are sequestered as dust grains condense from the gas, they ignore the detailed physics of grain nucleation and growth as well as the physical changes, for example, large-scale pulsations, thermal pulses, dredge-up of material from the stellar core to the surface, that the star itself can undergo.

A much more detailed description of chemical equilibrium in O-rich, C-rich and S-type (where $\mathrm{C} / \mathrm{O} \sim 1$ ) $\mathrm{AGB}$ stars that takes into account both gas-phase and condensed-phase species among 34 elements has been presented by Agúndez et al. ${ }^{16}$. Their detailed predictions for molecular abundances within $5 \mathrm{R}_{*}$, calculated through mimimising the total Gibbs free energy of the mixture, are compared to observational determinations where these are available. They show excellent agreement for many species, including $\mathrm{C}_{2} \mathrm{H}_{2}, \mathrm{HCN}, \mathrm{CS}, \mathrm{SiS}$, $\mathrm{SiO}, \mathrm{SiC}_{2}, \mathrm{HCP}, \mathrm{HF}$ and $\mathrm{HCl}$ in C-rich stars. For O-rich AGB stars, they find such agreement for $\mathrm{H}_{2} \mathrm{O}, \mathrm{CO}_{2}, \mathrm{H}_{2} \mathrm{~S}, \mathrm{SO}, \mathrm{SiO}$ and $\mathrm{PO}$. Many inorganic species are also observed in agreement with predictions in the inner envelope, including cyanides and isocyanides such as $\mathrm{MgCN}, \mathrm{MgNC}, \mathrm{NaCN}, \mathrm{KCN}$ and $\mathrm{CaNC}$, halides such as $\mathrm{AlF}, \mathrm{AlCl}, \mathrm{NaCl}$ and $\mathrm{KCl}$, and oxides such as $\mathrm{TiO}, \mathrm{TiO}_{2}$ and $\mathrm{AlO}$.

Agúndez et al. ${ }^{16}$ also note some significant failures in of the modelling, most importantly for the hydrides $\mathrm{H}_{2} \mathrm{O}, \mathrm{SiH}_{4}$ and $\mathrm{PH}_{3}$ in C-rich stars and $\mathrm{NH}_{3}$ in both C-rich and O-rich stars. The differences are very large, with calculated abundances falling some five to six orders of magnitude below those observed. Such failings have led to suggestions for the formation of these species through non-LTE chemistry, including shock and surface chemistry as discussed below. In addition to including simple diatomic and triatomic species, they have also used Density Functional Theory to calculate the thermochemical data for $\operatorname{Ti}_{x} \mathrm{C}_{y}$ species up to $x$ $=13$ and $y=22$ to identify the key condensation nuclei of the titanium carbide particles often found at the centre of extra-solar carbonaceous dust grains. 


\section{B. Pulsational Shock Waves}

Although LTE chemistry is treated in a 'steady-state' fashion, AGB stars undergo convective motions, large-scale pulsations and thermal pulses, all of which can lead to spatial and temporal inhomogeneities in physical conditions and to deviations from LTE abundances as well as to isotopic compositions. The effects of pulsational shocks on the LTE abundances have been studied in detail in a series of papers by Cherchneff and co-workers ${ }^{17-19}$. These periodic shocks drive large changes in both density and especially in temperature which essentially destroy all LTE molecules and allow a new gas-phase chemistry in the cooling post-shock gas. In addition, these dense cooling flows also allow dust grains to nucleate and grow. The shocked gas follows a ballistic trajectory, it is subject to repeated shocks, rising up and falling back in the atmosphere, thus enhancing the time-scale over which dust can grow over several pulsation cycles before radiation pressure on the grains eventually enables both the gas and the dust to escape to the circumstellar envelope. The composition of this gas can be significantly different from that predicted by the LTE models.

In particular, Cherchneff ${ }^{17}$ has shown that hot water can be produced at an abundance close to that observed in IRC +10216 from $\mathrm{O}$ atoms released in the collisional destruction of $\mathrm{CO}$ in the hot post-shock gas.

\section{Dust Formation}

The formation of dust grains in AGB stars has traditionally been described by nucleation theory in which grains grow by the addition of monomers to seed particles and assumes chemical equilibrium ${ }^{20-22}$. More recently, there have been attempts to describe the kinetic growth of dust grains, or more precisely, grain seeds, through using quantum mechanical calculations to determine the lowest energy structures and pathways to growth. Thus, Goumans and Bromley ${ }^{23}$ performed DFT calculations to identify the lowest energy pathway from $\mathrm{SiO}$ to $\mathrm{Mg}_{4} \mathrm{Si}_{2} \mathrm{O}_{9} \mathrm{H}_{2}$, through the successive additions of either an oxygen or an $\mathrm{Mg}$ or Si atom at each intermediate step. Their calculations showed a common problem on forming grains, namely that the first steps in growth are often highly endoergic. Similar approaches have been made by Bromley et al. ${ }^{24}$, Gobrecht et al. ${ }^{25}$ and Boulangier et al. ${ }^{26}$ who combine DFT calculations with a chemical kinetic theory of growth and a new approach 
to nucleation.

\section{Gas-Grain Chemistry}

With the exception of grain formation at very high temperatures, gas-grain chemistry has been neglected in essentially all circumstellar chemical models to date. This neglect is due to the fact that the time-scales for collisions of the gas with the dust is shorter than the expansion time-scale only at radial distances where the grains are warm enough that few species can remain bound to their surfaces. Observations do indicate, however, that the gas-grain interaction may be significant even in the warm $(\mathrm{T} \sim 100-300 \mathrm{~K})$ inner regions of the outflow. Thus, infrared observations of ro-vibrational transitions have shown that fully hydrogenated species such as $\mathrm{CH}_{4}, \mathrm{NH}_{3}, \mathrm{C}_{2} \mathrm{H}_{4}$, and $\mathrm{SiH}_{4}^{2,27,28}$, have abundances that appear to increase at radial distances of around 10-40 stellar radii in IRC+10216. In the interstellar medium, large abundances of fully saturated molecules are associated with Hot Molecular Cores, regions of high mass star formation with temperatures in the range 100-300 $\mathrm{K}$ and $\mathrm{H}_{2}$ number densities of $10^{6}-10^{8} \mathrm{~cm}^{-3}$. Such high abundances result from the desorption of ice mantles in which these hydrides have formed through hydrogen atom addition to $\mathrm{C}, \mathrm{N}$ and $\mathrm{O}$ atoms on the ice surface. Although there is no evidence that C-rich AGB envelopes have ice-covered mantles, it is possible that the formation of these hydrides could be the result of reactions involving chemisorbed species on warm, bare carbonaceous grains. The availability of chemisorption sites on bare grains may also serve as a trap for gas-phase molecules and radicals and lead to the removal of species from the gas. In particular, molecules involving silicon, such as $\mathrm{SiO}, \mathrm{SiC}, \mathrm{SiS}, \mathrm{SiC}_{2}$, and $\mathrm{Si}_{2} \mathrm{C}$, are often seen to have gas-phase abundances that decrease in this region of the $\mathrm{CSE}^{29-35}$.

Van de Sande et al. ${ }^{36}$ have recently produced the first model of the circumstellar chemistry that includes the gas-grain interaction, with applications to both C-rich and O-rich envelopes. Their model includes the accretion of gas-phase species on to the dust grains together with surface chemistry involving hydrogenation and reactions between atoms and radicals. The ice mantles that form can be removed through thermal desorption, photodesorption and sputtering of ice with abundant gas-phase molecules due to differential velocities in the outflow between the gas and the dust particles. The authors find that abundances can be significantly altered in some circumstances depending on the choice of model param- 
eters: mass-loss rate and expansion velocity - increasing $\dot{M} / v_{e}$ increases the density in the CSE and thereby shortens the collisional time between gas and grains; grain temperature - which depends on the material composition, size and morphology of the dust particles. Surface chemistry is enhanced by faster diffusion rates on warmer grains; and drift velocity - sputtering rates increase as this increases. Although the binding energies of the gas-phase species are taken from laboratory studies of those in water ice mantles rather than on bare carbonaceous or silicate materials, these models are an important first step in considering the interaction of gas and dust in CSEs. Subsequently, Van de Sande et al. ${ }^{37}$ considered the effects of different grain size distributions and performed radiative transfer calculations in order to compare with molecular line observations finding that, in general terms, a combination of their gas-grain model with observation may be able to constrain the size distribution of dust grains in CSE envelopes.

\section{E. The Outer CSE}

Chemistry in the outer region of the CSE is dominated by radiation chemistry, that is, by the interaction of the outflowing wind with the external UV radiation field produced by the galactic stars in its neighbourhood. Since this interaction generates reactive radicals and ions, it is important to treat the photodissociation of abundant molecules as accurately as possible. In recent years, important advances in this regard have been made in accounting for the self-shielding of $\mathrm{CO}^{38}$ and $\mathrm{N}_{2} \cdot{ }^{39,40}$

Given the significance of photons to determining the abundances and radial distributions of molecules, we devote the following Section to the important topic of photon-driven chemistry.

\section{PHOTOCHEMISTRY}

\section{A. External (Interstellar) UV Photons}

Many of the molecules detected through micro- and millimeter-wave rotational spectroscopy show clumpy, shell-like distributions on angular sizes of 10-20 arcsec. These distributions have been interpreted, rather successfully, as arising from the photochemistry induced by the destruction of outflowing, or 'parent', molecules from the inner CSE by in- 
coming interstellar UV radiation. For stars such as IRC +10216 , an extinction at visible wavelengths of $A_{V}=1 \mathrm{mag}^{41}$ falls in a region of high density, $\mathrm{n} \sim 10^{5} \mathrm{~cm}^{-3}$ so that radicals and ions produced by UV photons collide and react to build more complex species on short time scales. Millar Millar et al. ${ }^{11}$ showed that a photochemical model could explain both the decreasing column densities of the cyanopolyynes $\left(\mathrm{HC}_{2 n+1} \mathrm{~N}, n=1-4\right)$ and the increasing radii of their peak abundances as their size increased. Such models, which assume a spherically symmetric CSE at constant mass-loss rate and outflow velocity, were very successful but do not address some of the more recent evidence that the mass-loss rate is not in steady state nor symmetric. For example, Mauron and Huggins ${ }^{42}$ showed that the CSE of IRC +10216 contains a series of high density shells, while high spatial resolution observations of the cyanopolyynes and other hydrocarbon chains show that the molecular gas in these shells is clumped.

Brown and Millar ${ }^{43}$ and Cordiner and Millar ${ }^{44}$ considered a simple model for these density-enhanced shells and showed that they has an impact on the chemistry due to both shorter collisional time scales and enhanced extinction against UV photons within the shells. The first 'clumpy' model of the outflow was developed to explain the detection of hot water in IRC $+10216,{ }^{13}$ mentioned above in Sect. I. This model essentially allows a few percent of interstellar UV photons to penetrate deep into the molecular CSE and dissociate ${ }^{13} \mathrm{CO}$ and $\mathrm{SiO}$ unimpeded by the $40 \mathrm{mag}$ or so of dust extinction at UV wavelengths, appropriate for a mass-loss rate of $2 \times 10^{-5} \mathrm{M}_{\odot} \mathrm{yr}^{-1}$ at a radius of $2 \times 10^{15} \mathrm{~cm}$. The liberated $\mathrm{O}$ atoms then react quickly with $\mathrm{H}_{2}$ at these warm temperatures to form $\mathrm{H}_{2} \mathrm{O}$. The radical $\mathrm{OH}$, the photodissociation product of water is also detected in $\mathrm{IRC}+10216^{45}$. Note that the abundant ${ }^{12} \mathrm{CO}$ species is not photodissociated since it self-shields very efficiently ${ }^{38}$.

Our detailed chemical kinetic models of the chemistry in the CSEs use the codes and reaction rate coefficients ${ }^{46}$ publicly available from the UMIST Database for Astrochemistry website ${ }^{47}$, with updated photodissociation rates calculated using the cross-sections ${ }^{48}$ provided in the Leiden photochemistry database ${ }^{49}$. The initial, or parent, molecular abundances, that is for those molecules which are formed close to the stellar surface, are taken from Agúndez Agúndez et al. ${ }^{13}$ and shown in Table I. In this paper, we shall consider fractional abundances of species $i$ as a function of radial distance $r, x_{i}(r)=n_{i}(r) / n_{H_{2}}(r)$, where we note that the $\mathrm{H}_{2}$ molecule is not photodissociated by the incident radiation field, so that $n_{H_{2}}(r)$ is proportional to $r^{-2}$ for an envelope expanding at constant velocity $v_{e}$. 
TABLE I. Parent species and their initial fractional abundances relative to $\mathrm{H}_{2}$.

\begin{tabular}{|c|c|c|c|}
\hline Species & Abund & Species & Abund \\
\hline $\mathrm{He}$ & $1.7 \times 10^{-1}$ & $\mathrm{CO}$ & $8.0 \times 10^{-4}$ \\
\hline $\mathrm{N}_{2}$ & $4.0 \times 10^{-5}$ & $\mathrm{C}_{2} \mathrm{H}_{2}$ & $8.0 \times 10^{-5}$ \\
\hline $\mathrm{HCN}$ & $2.0 \times 10^{-5}$ & $\mathrm{SiS}$ & $1.0 \times 10^{-6}$ \\
\hline $\mathrm{SiO}$ & $1.2 \times 10^{-2}$ & $\mathrm{CS}$ & $5.0 \times 10^{-7}$ \\
\hline $\mathrm{SiC}_{2}$ & $5.0 \times 10^{-8}$ & $\mathrm{HCP}$ & $2.5 \times 10^{-8}$ \\
\hline
\end{tabular}

We can solve for the fractional abundance, $x_{i}(r)$, of species $i$ as a function of radius. The ODE that describes the evolution of the fractional abundance of $i$ due to both the expansion and chemistry can be written as:

$$
\frac{\mathrm{d} x_{i}}{\mathrm{~d} r}=\frac{1}{v_{e}}\left[\sum_{j, k} k_{j k} x_{j} x_{k} n_{H_{2}}(r)+\sum_{l} k_{l} x_{l}-x_{i}\left[\sum_{m} k_{i m} x_{m} n_{H_{2}}(r)+\sum_{n} k_{n}\right]\right]
$$

The first and third terms on the right hand side are the summation over all two-body reactions leading the formation and destruction of species $i$, respectively, while the second and fourth terms represent one-body reactions, that is photo-processes and cosmic-ray ionisations, leading to the formation and destruction of $i$, respectively.

We assume that the radial distribution of the gas temperature is given by a power-law of the form $T(r)=T_{\text {star }}\left(r / R_{\text {star }}\right)^{p}$, where $T_{\text {star }}$ and $R_{\text {star }}$ are the stellar temperature and radius, respectively, and $p=-0.7$.

Fig. 1 shows the radial distribution of water and $\mathrm{OH}$ for both a smooth flow and for the 'low extinction-type' model proposed by Agúndez et al. ${ }^{13}$ for parameters typical of IRC +10216 , i.e. $\dot{M}=2 \times 10^{-5} \mathrm{M}_{\odot} \mathrm{yr}^{-1}$ and $v_{e}=14.5 \mathrm{~km} \mathrm{~s}^{-1}$. In the latter case I adopt the ad hoc assumption of Agúndez et al. ${ }^{13}$ that $2.5 \%$ of interstellar UV photons are not extincted by dust and can penetrate down to the innermost envelope with the remaining $97.5 \%$ of photons extinguished by dust. The results confirm those of Agúndez et al. For the standard smooth model, although water can be made in the internal CSE, that is, at radii less than $10^{16} \mathrm{~cm}$, its abundance is more than two orders of magnitude less than that observed. The clumpy model on the other hand efficiently photodissociates the initial abundance of $\mathrm{SiO}$ and converts the oxygen atoms released into $\mathrm{H}_{2} \mathrm{O}$ at an abundance which agrees with the range derived from observation ${ }^{50},(4-24) \times 10^{-7}$. Note that the 
photodissociation rate adopted for $\mathrm{SiO}$ at $10^{14} \mathrm{~cm}$, the start of the outflow, is equivalent to an effective UV extinction of 3.7 in the clumpy medium, much less than the its value of about 420 in a smooth outflow.

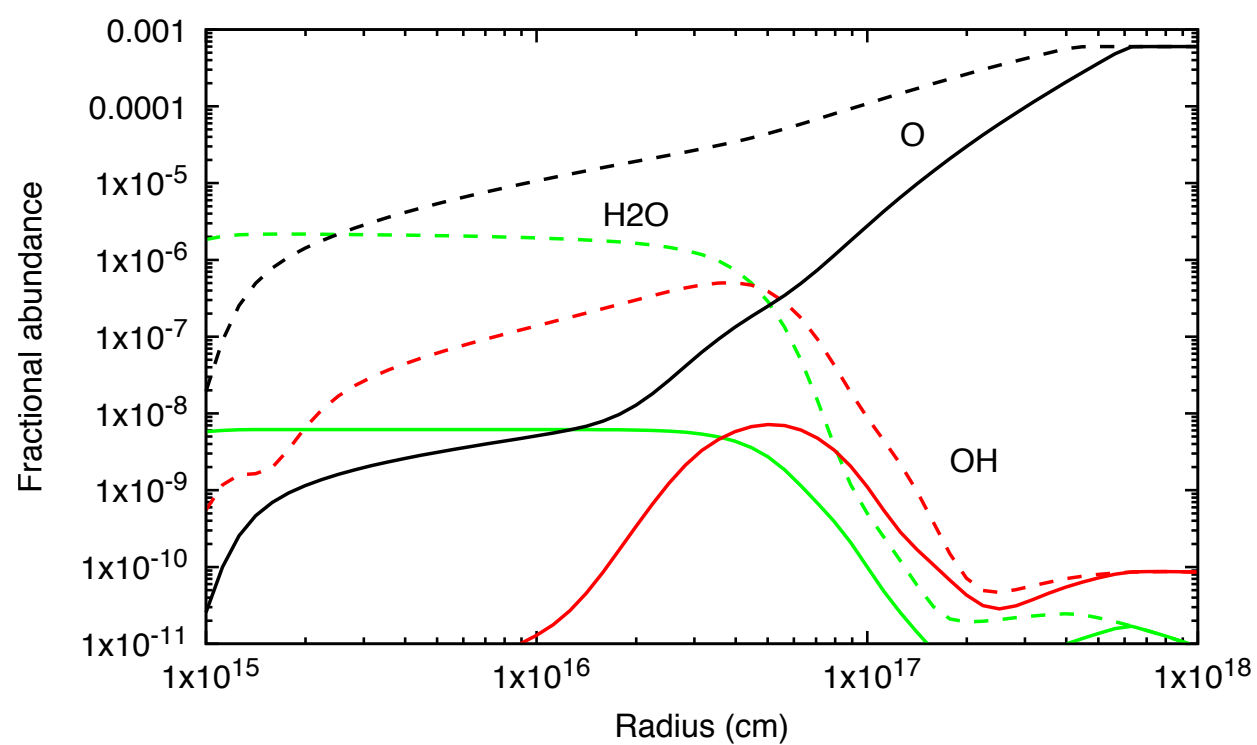

FIG. 1. Molecular abundances relative to $\mathrm{H}_{2}$ as a function of radius in a model of $\mathrm{IRC}+10216$ that assumes $2.5 \%$ of interstellar UV photons are able to penetrate to $10^{15} \mathrm{~cm}$ without being extinguished by dust grains. The solid curves refer to a 'smooth' outflow, that is one in which the density distribution follows a $1 / r^{2}$ distribution. Dashed lines refer to the clumpy model. Colour code: Green, $\mathrm{H}_{2} \mathrm{O}$; Red, OH; Black, O.

This simple model was improved by Van de Sande Van de Sande et al. ${ }^{51}$ who investigated the role of clumps and pores on the transfer of interstellar photons to the inner CSE. Instead of an ad hoc assumption of the fraction of photons able to be transported free of the effects of extinction, they assumed that the CSE is composed of a size distribution of clumps that takes up a fraction $f_{v o l}$ of the CSE and which are embedded in an interclump medium characterised by the parameter $f_{i c}=\rho_{i c} / \rho_{s m}$, the ratio of the interclump density to that of a smooth, uniform outflow. Thus, $f_{i c}=1$ is equivalent to a smooth outflow, while $f_{i c}=0$ represents a void interclump medium, that is a one-component outflow.

Van de Sande Van de Sande et al. ${ }^{51}$ calculate an effective UV optical depth for external, interstellar photons, $\tau_{e f f}$, from radial distance $r$ to infinity for an exponential distribution 
of clumps,

$$
f(\tau)=\frac{1}{\tau_{c l}} \exp \left(-\tau / \tau_{c l}\right)
$$

where $\tau_{c l}$ is the average optical depth of the clumps. This approach was applied to the chemistries of both O-rich and C-rich CSEs over a range of mass-loss rates from $10^{-7}$ to $10^{-5} \mathrm{M}_{\odot} \mathrm{yr}^{-1}$.

Fig. 2 shows the abundance distributions when the approach by Van de Sande Van de Sande et al. ${ }^{51}$ is taken for the same physical parameters as used in Fig. 1. The particular case shown here is a one-component model, which minimises the dust extinction seen by interstellar photons, with $f_{\text {vol }}=0.1, f_{i c}=0$, that is where the mass of the outflow is contained in clumps that take up one-tenth of the total volume. Note that the calculations presented by Van de Sande et al. ${ }^{51}$ contained an error in that CO self-shielding was not included - see the correction published by Van de Sande et al. ${ }^{52}$. This has a large effect on abundances in the inner envelopes of the one-component flows at the highest mass-loss rates, $10^{-5} \mathrm{M}_{\odot} \mathrm{yr}^{-1}$. In particular, the large enhancements derived by Van de Sande Van de Sande et al. ${ }^{51}$ for $\mathrm{HCN}$ in the O-rich case and for $\mathrm{H}_{2} \mathrm{O}$ in the C-rich case have been over-estimated. Note also that the effects for lower mass-loss rates are less sensitive to this omission since column densities of $\mathrm{CO}$ are reduced and therefore less sensitive to the effects of self-shielding in these cases. Fig. 2 does, however, include the correct CO self-shielding, although the Van de Sande models do not consider that of $\mathrm{N}_{2}$, an omission that may affect their conclusions to some degree, particularly for lower mas-loss rates. One sees here that, for this mass-loss rate, $2 \times 10^{-5} \mathrm{M}_{\odot} \mathrm{yr}^{-1}$, and volume filling factor, the radial distribution of the water abundance is essentially identical for both the smooth and clumped outflows and much less than its observed value.

Fig. 3 shows, again for a one-component model, that if the outflow is extremely clumpy, $f_{\text {vol }}=0.01$, then external photons can penetrate to the inner CSE, at a radial distance of less than $10^{16} \mathrm{~cm}$, and increase the water abundance by a factor of a few and the $\mathrm{OH}$ abundance by several orders of magnitude. It is noticeable that the radial distribution of $\mathrm{OH}$ moves inward in this case due to the increased photodissociation rate of $\mathrm{H}_{2} \mathrm{O}$ in the inner envelope. However, such small filling factors are inconsistent with the observations of IRC+10216. 


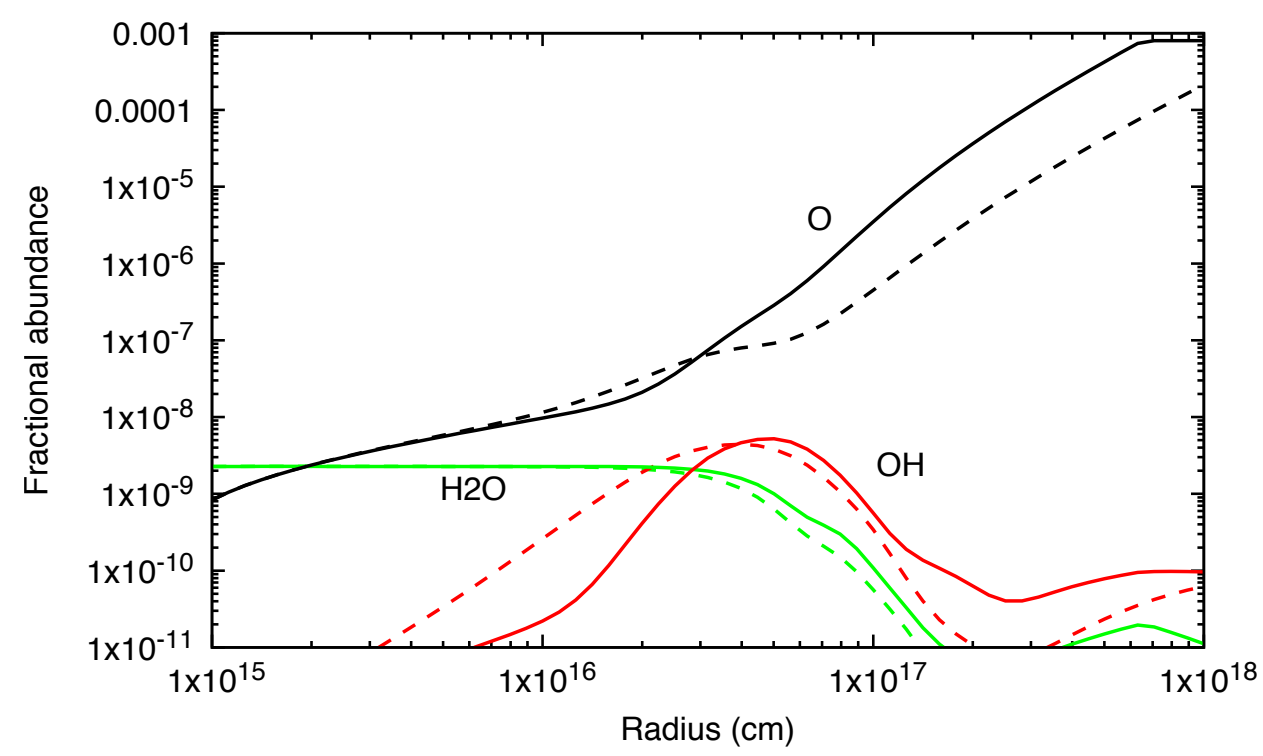

FIG. 2. As Fig. 1, except for a porous, clumpy, mass distribution in the CSE. In particular, these results are for a one-component distribution of mass, taking up a fraction 0.1 of the total volume of the CSE. The solid curves refer to the smooth flow, dashed curves to the one-component model. Colour code: Green, $\mathrm{H}_{2} \mathrm{O}$; Red, OH; Black, O.

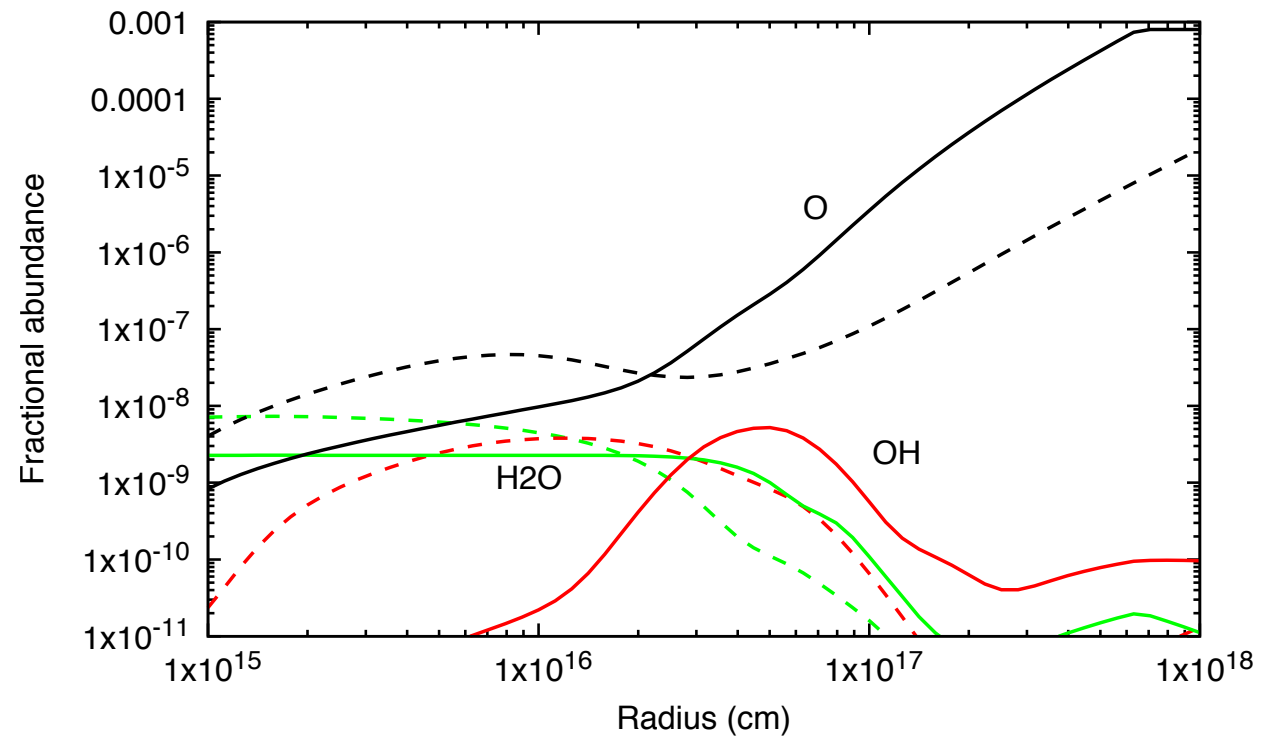

FIG. 3. As Fig. 2, except that the results are for a one-component distribution of mass, taking up a fraction $f_{v o l}=0.01$ of the total volume of the CSE. The solid curves refer to the smooth flow, dashed curves to the one-component model. Colour code: Green, $\mathrm{H}_{2} \mathrm{O}$; Red, OH; Black, O. 


\section{B. Internal (Stellar) UV Photons}

Since AGB stars are cool, with effective temperatures generally less than $3000 \mathrm{~K}$, and form copious amounts of dust, it was rarely considered that they might emit sufficient UV

photons to affect chemistry. Millar ${ }^{53}$ was the first to suggest that internally generated stellar (blackbody) photons might affect the chemistry of the internal CSE of IRC+10216 $\left(T_{\text {eff }}=\right.$ $2330 \mathrm{~K}$ ). At $50 \mathrm{R}_{*}$ (or about 1 arcsec at the distance of IRC+10216), the flux of unshielded stellar photons falls to more than 100 times less than the interstellar UV flux for $\lambda<$ $1600 \AA$, so that many species, including $\mathrm{CO}$ and $\mathrm{N}_{2}$, are unaffected by the presence of these photons. Nevertheless, in a clumpy medium, internal stellar photons can play a significant role in driving a selective chemistry on the spatial scales now being probed by the ALMA interferometer.

For a smooth outflow, the radial extinction in the UV from radius $r$ to infinity is proportional to $1 / r$. Thus, for the cool AGB stars, internal photons have a very large dust extinction as they propagate outwards and play no, or at most a very limited, role in chemistry for mass-loss rates above $10^{-7} \mathrm{M}_{\odot} \mathrm{yr}^{-1}$. If the flows are porous, however, internal photons can find pathways of low extinction to the external CSE although their effects are small since, in addition to dust extinction, the photon flux falls off as $1 / r^{2}$ due to geometric dilution.

Van de Sande and Millar ${ }^{54}$ applied the porosity formalism to investigate the effects of internal stellar photons on chemistry. They found that for mass-loss rates above $10^{-6} \mathrm{M}_{\odot}$ $\mathrm{yr}^{-1}$, the very large effective UV optical depths that occur between the dust formation zone and around $10 \mathrm{R}_{*}$, that is within a radial distance of about $3-5 \times 10^{14} \mathrm{~cm}$, means that there is no effective photochemistry. For smaller mass-loss rates, however, smaller effective optical depths mean that these photons can affect chemistry, again in a selective manner, and with the largest effects seen in the one-component models which have the lowest optical depths. Thus, a point at radius $r$ from the star sees external UV photons at an optical depth $\tau_{\text {eff }}(r)$ and stellar UV photons at an optical depth,

$$
\Delta \tau_{e f f}(r)=\tau_{e f f}\left(r_{d}\right)-\tau_{e f f}(r)
$$

where $\tau_{\text {eff }}\left(r_{d}\right)$ is the optical depth from the dust formation radius, $r_{d}$, to infinity.

Fig. 4 shows the effective extinction in the UV for internal photons propagating outwards from the star for a variety of mass-loss rates for smooth, one-component and two-component 
TABLE II. Model parameters.

\begin{tabular}{llll}
\hline Species & Abund & Species & Abund \\
\hline$\dot{M}$ & $2.0 \times 10^{-5} \mathrm{M}_{\odot} \mathrm{yr}^{-1} \mathrm{v}_{e}$ & $14.5 \mathrm{~km} \mathrm{~s}^{-1}$ \\
$\mathrm{~T}_{\text {star }}$ & $2330 \mathrm{~K}$ & $\mathrm{R}_{\text {star }}$ & $5.0 \times 10^{13} \mathrm{~cm}$ \\
$\mathrm{R}_{\text {dust }}$ & $9.5 \times 10^{13} \mathrm{~cm}$ & $\mathrm{p}$ & -0.7 \\
No. Reactions & 6516 & No. Species 468
\end{tabular}

(weighted) models. The appropriate rates for photodestruction by internal photons are calculated using the cross-sections from Heays et al. ${ }^{48}$ as:

$$
\beta_{i}(r)=\beta_{i}\left(r_{s c}\right)\left(\frac{r_{s c}}{r}\right)^{2} \exp \left(-\gamma_{i} \Delta A_{V}(r)\right)
$$

where $\beta_{i}\left(r_{s c}\right)$ is the unshielded photorate of species $i$ at a scaling radius $r_{s c}$, and $\Delta A_{V}(r)$ is the visual extinction from the dust formation radius to radial distance $r$. For those species for which cross-sections are unknown, we use the scaling formula commonly used in astrochemical studies of interstellar clouds in which the unshielded interstellar rate, $\beta_{0}^{\text {IS }}$, is multiplied by the ratio of the integrated fluxes of stellar to interstellar photons (integrated over $6-13.6 \mathrm{eV})$ :

$$
\beta_{0}^{\mathrm{IP}}=\left(\frac{G_{*}}{G_{\mathrm{IS}}}\right) \beta_{0}^{\mathrm{IS}}
$$

where

$$
G=\int_{2068 \AA}^{912 \AA} F(\lambda) \mathrm{d} \lambda
$$

and $F(\lambda)$ is the photon flux.

This approach can significantly overestimate the photoionisation rates for molecules since their ionization potentials generally fall at energies where the flux of stellar UV photons depends sensitively on the assumed blackbody temperature of the source. For these species we have used a reduction factor determined from comparing photoionisation rates calculated for atomic ionisation, for which the exact cross-sections are known, to those calculated using the integrated approach. We include 336 photochannels due to stellar internal photons. Our total chemical kinetic network thus includes, when internal photons from a companion object (see next Section) are also included, some 6516 reactions among 468 species.

Table II gives the stellar parameters adopted for the models in this Section and Fig. 5 shows the results for a model similar to that in Fig. 2 with the addition of stellar photons at 


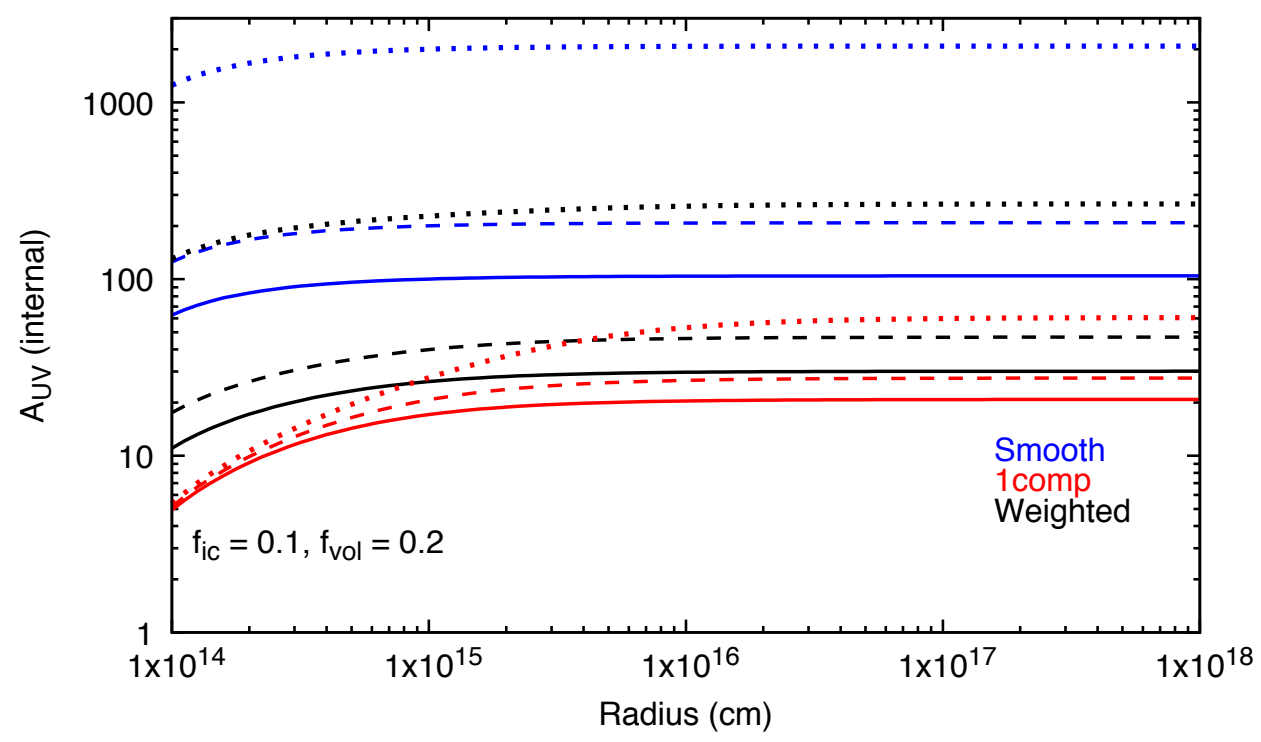

FIG. 4. Effective radial extinction in magnitudes of internally generated UV photons for mass-loss rates of $10^{-6} \mathrm{M}_{\odot} \mathrm{yr}^{-1}$ (solid lines), $2 \times 10^{-6} \mathrm{M}_{\odot} \mathrm{yr}^{-1}$ (dashed lines), and $2 \times 10^{-5} \mathrm{M}_{\odot} \mathrm{yr}^{-1}$ (dotted lines). The cases of smooth, one-component and two-component (weighted) outflows are color coded.

a blackbody temperature of $2330 \mathrm{~K}$, the effective temperature of IRC+10216. In this case, we see that the internal, stellar photons do not play any role in increasing the abundance of water. Such low-energy photons can affect molecular distributions for smaller mass-loss rates and Fig. 6 shows the distributions for $\dot{M}=10^{-6} \mathrm{M}_{\odot} \mathrm{yr}^{-1}$ and an extremely clumped outflow with $f_{\text {vol }}=0.01$. Here one sees an enhancement in the fractional abundance of water by about an order of magnitude at $10^{15} \mathrm{~cm}$. One should note that the amount of dust and hence extinction is directly proportional to the mass-loss rate. Thus, at radius $r$, both the total and effective extinctions are an order of magnitude less in Fig. 6 as those in Figs. 2 and 5. As a result, the distributions of water lie closer to the star in the case of lower mass-loss rate since interstellar photons do not suffer as much extinction while stellar blackbody photons also suffer less extinction as they propagate outwards through the CSE.

\section{Internal (Companion) UV Photons}

Stellar blackbody photons may not be the only source of internal UV photons in some AGB stars. Broadband GALEX observations show that a significant fraction of AGB stars 


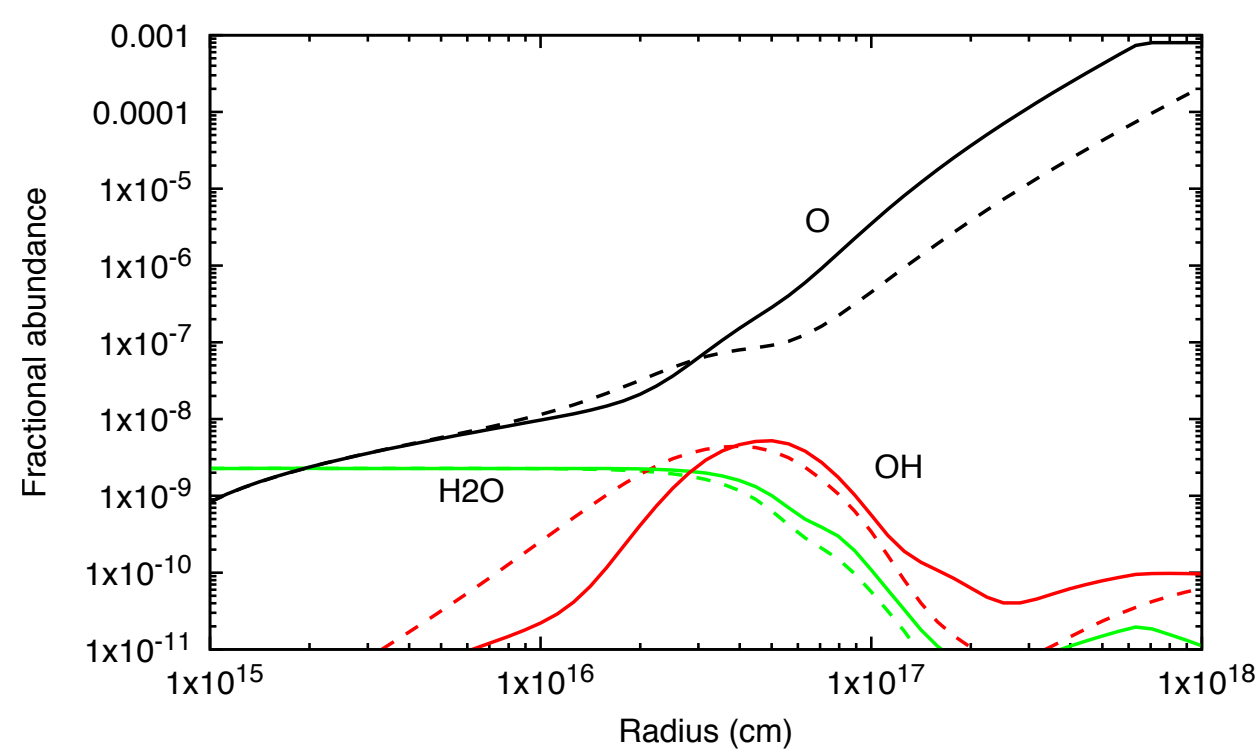

FIG. 5. Water abundance in a model of IRC +10216 that assumes a porous, one component, clump mass distribution in the CSE with $f_{v o l}=0.1$ and includes a source of internal UV photons from a stellar blackbody at a temperature of $2330 \mathrm{~K}$. The solid curves refer to the smooth flow, dashed curves to the one-component model. Colour code: Green, $\mathrm{H}_{2} \mathrm{O}$; Red, OH; Black, O.

are detectable at UV wavelengths. In a survey of 316 AGB stars, Montez Montez et al. ${ }^{55}$

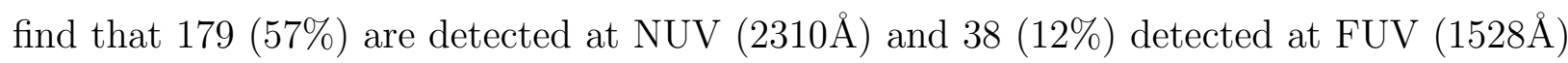
wavelengths. In a careful study, they show that de-reddened, distance-corrected NUV fluxes correlate inversely with $\dot{M} / v_{e}$, a measure of the $\mathrm{H}_{2}$ number density per unit volume in the CSE, and that in some cases the UV fluxes correlate with the optical light curves, evidence consistent with a stellar, or intrinsic, source for the UV radiation.

In a similar manner, Ortiz and Guerrero ${ }^{56}$ have shown that main-sequence binary companions of AGB stars can be inferred from the detection of an AGB star in the GALEX far-UV band and with an observed flux ratio more than 20 times that predicted in the GALEX near-UV band. They show that 34 stars out of a volume-limited sample of 58 fulfill these criteria indicating that they have a main-sequence companion earlier than spectral type K0. Furthermore, they argue that the excess UV emission is not due to a single temperature companion, as might be expected from a star, but may reflect either absorption by the extended atmosphere and CSE of the AGB star or be produced by an accretion flow. Subsequently, Ortiz et al. ${ }^{57}$ argued from a survey of some 20 UV-emitting AGB stars that the far-UV, i.e. the highest energy, photons might arise from a hot companion or an 


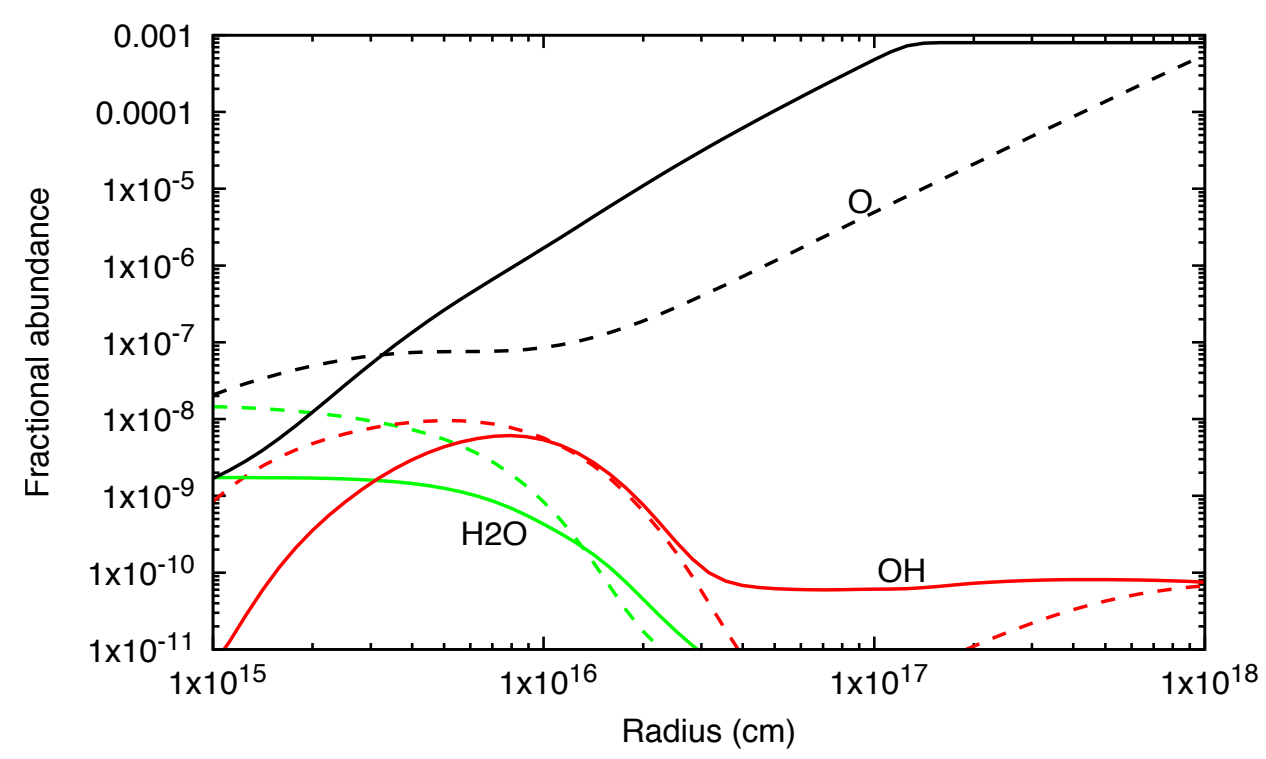

FIG. 6. As Fig. 3, but for a mass-loss rate of $10^{-6} \mathrm{M}_{\odot} \mathrm{yr}^{-1}$ and a source of internal UV photons from a stellar blackbody at a temperature of $2330 \mathrm{~K}$. Here $f_{v o l}=0.01$. The solid curves refer to the smooth flow, dashed curves to the one-component model. Colour code: Green, $\mathrm{H}_{2} \mathrm{O}$; Red, $\mathrm{OH}$; Black, O.

accretion disk.

These results indicate that internal UV radiation may be present in a significant fraction of AGB stars, generated either by a stellar companion or by the accretion disk around a star or a planet.

The physical effects of companion objects may also be imprinted on the density structure of the CSE gas. Thus the beautiful spiral patterns detected in IRC $+10216^{58}$ and $\mathrm{R}$ Sculptoris ${ }^{59}$, LL Peg ${ }^{60}$ and the equatorial density enhancement in $\mathrm{L}_{2} \mathrm{Pup}^{61,62}$, as well as the complex density structures seen in all O-rich AGB stars studied at high spatial resolution in the ALMA Large Programme ATOMIUM ${ }^{63}$, can all be interpreted in terms of an orbiting stellar or planetary companion that perturbs the radial outflow from the AGB star. Recently, Homan et al. ${ }^{64}$ used ALMA to study the CO and SiO emission in the CSE of the O-rich AGB star EP Aqr at high angular resolution. They found evidence of complex hydrodynamic flows in the inner envelope including a central equatorial density enhancement, which itself contains spiral structures, a bi-conical outflow and a spherical void in the $\mathrm{SiO}$ emission at a distance of $\sim 55$ au from the star. They argued that the void is due to UV destruction of $\mathrm{SiO}$ by a white dwarf companion at a temperature of around $6000 \mathrm{~K}$ and a mass 
between 0.65 and $0.8 \mathrm{M}_{\odot}$ and, furthermore, that it is this companion that perturbs nonradial motions in the wind of the AGB star. Several theoretical models have been presented showing that the nature of these complex structures depends on the radial momentum in the wind compared to the orbital angular momentum of the companion and therefore on parameters such as the mass-loss rate, the mass ratio of the primary and companion, the orbital radius of the companion, and the stellar wind velocity at the orbital distance of the companion, amongst others. ${ }^{65-67}$

In order to examine the effects of UV flux from a companion object, we show in Fig. 7 results from a series of representative models for a carbon-rich outflow that combine our porosity approach with three sources of UV radiation: (i) the external interstellar flux, (ii) a cool (2330 K) blackbody AGB stellar flux, as discussed in Sect.III B, and (iii) a hotter blackbody flux from a companion object such as a white dwarf or an accretion disk. In these models, we choose a white dwarf companion with temperature equal to $6000 \mathrm{~K}$ and a radius of $1.8 \times 10^{10} \mathrm{~cm}\left(0.26 \mathrm{R}_{\odot}\right)$. We note that, at this temperature, the neglect of $\mathrm{N}_{2}$ self-shielding does have an effect on the abundances calculated for $\mathrm{N}_{2}$ in the inner envelope for the one-component models. In particular, the models may severely underestimate the $\mathrm{N}_{2}$ abundance for radii less than a few times $10^{14} \mathrm{~cm}$, although its impact on the abundances of $\mathrm{C}^{+}$and $\mathrm{H}_{2} \mathrm{O}$, discussed below, is likely to be minimal. In these figures, we show the fractional abundances as a function of radius calculated for three models: green curves are for 'smooth' outflows, i.e. with density proportional to $r^{-2}$; red curves are for 'one-component' models in which the interclump density is zero, i.e. $f_{i c}=0$; and black curves represent the fractional abundance weighted over those calculated for the interclump and clumpy media. For this we use the formula provided by Van de Sande et $a l .{ }^{51}$ :

$$
x_{w t}(r)=x_{c l}(r)+f_{i c}\left(1-f_{v o l}\right)\left(x_{i c}(r)-x_{c l}(r)\right)
$$

where $x_{w t}, x_{c l}$ and $x_{i c}$ are the weighted, clump and interclump fractional abundances, respectively. These particular models are for the case of $f_{i c}=0.1$ and $f_{v o l}=0.2$ and for mass-loss rates of $10^{-6} \mathrm{M}_{\odot} \mathrm{yr}^{-1}, 2 \times 10^{-6} \mathrm{M}_{\odot} \mathrm{yr}^{-1}$, and $2 \times 10^{-5} \mathrm{M}_{\odot} \mathrm{yr}^{-1}$.

The impact of internal, companion UV photons is very significant in the case of low mass-loss rates with the entire CSE showing a significant degree of ionisation from the photo-degradation of parent $\mathrm{C}_{2} \mathrm{H}_{2}, \mathrm{HCN}$ and $\mathrm{CH}_{4}$. In these cases it can be seen that, even for mass-loss rates on the order of $2 \times 10^{-5} \mathrm{M}_{\odot} \mathrm{yr}^{-1}$, abundant $\mathrm{C}$ and $\mathrm{C}^{+}$can be formed just 

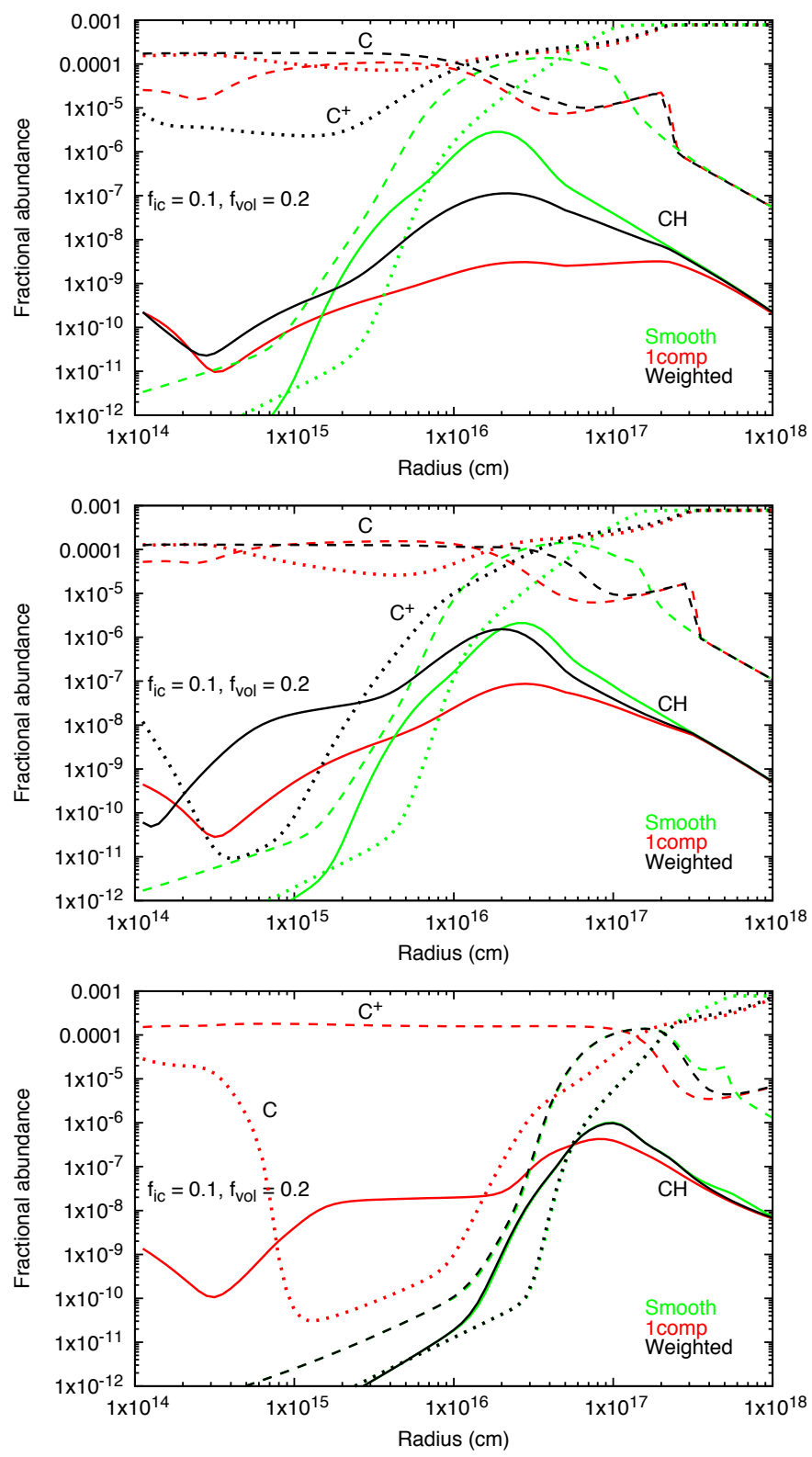

FIG. 7. Distribution of $\mathrm{C}, \mathrm{C}^{+}$and $\mathrm{CH}$ fractional abundances for a stellar $\mathrm{BB}$ at $2330 \mathrm{~K}$ and a white dwarf companion at $6000 \mathrm{~K}$. Mass-loss rates are $10^{-6} \mathrm{M}_{\odot} \mathrm{yr}^{-1}\left(\right.$ top), $2 \times 10^{-6} \mathrm{M}_{\odot} \mathrm{yr}^{-1}$ (middle), and $2 \times 10^{-5} \mathrm{M}_{\odot} \mathrm{yr}^{-1}$ (bottom). Solid lines show the $\mathrm{CH}$ abundance, dashed lines that of $\mathrm{C}$, and dotted lines $\mathrm{C}^{+}$.

outside the dust formation zone, although the latter's abundance falls rapidly as internal photons from the white dwarf get extinguished by dust grains. These species are due to the photodissociation of parent $\mathrm{HCN}$ and $\mathrm{C}_{2} \mathrm{H}_{2}$ with $\mathrm{C}^{+}$most abundant in the highly clumped, one-component outflows with $f_{\text {vol }}=0.2$ (Fig. 7). As $f_{\text {vol }}$ increases, the outflow becomes 
'smoother' $\left(f_{\text {vol }}=1\right)$ and the ability of internal photons to produce $\mathrm{C}^{+}$decreases ('smooth' curves, Fig. 7).

Finally, we discuss the effect of companion UV photons on the radial distribution of water, which we discussed previously in Sec. III A and Sec. III B. In both cases we found that the fractional abundance of $\mathrm{H}_{2} \mathrm{O}$ increased somewhat depending on the value of the mass-loss rate and the degree of porosity with the largest effect seen in the one-component models at low values of $\dot{M}$. Fig. 8 shows the distribution of $\mathrm{H}_{2} \mathrm{O}, \mathrm{OH}$ and $\mathrm{O}$ for mass-loss rates of $10^{-6}$ $\mathrm{M}_{\odot} \mathrm{yr}^{-1}, 2 \times 10^{-6} \mathrm{M}_{\odot} \mathrm{yr}^{-1}$, and $2 \times 10^{-5} \mathrm{M}_{\odot} \mathrm{yr}^{-1}$ with an additional companion UV field from a $6000 \mathrm{~K}$ blackbody.

Unlike the cases discussed earlier in Sec. III B, in which internal radiation is provided solely by a cool stellar blackbody, the addition of an internal $6000 \mathrm{~K}$ radiation field from a white dwarf companion destroys $\mathrm{H}_{2} \mathrm{O}$ in the inner regions and its abundance is much less, by more than an order of magnitude, than that found when the companion field is absent (see Fig. 5) and much less than its observed fractional abundance of $\sim 10^{-7}$.

\section{CONCLUSIONS}

Models of the chemical processes in the circumstellar envelopes of AGB stars that adopt spherical symmetry are still important in describing the observation of molecular emission in the outer envelopes where the majority of molecules are found. It is clear, however, that the density distributions of the inner CSEs, in particular, can no longer be described in terms of spherically symmetric, constant velocity outflows irradiated solely by external interstellar UV photons. Furthermore, the fact that AGB stars are cool, with effective temperatures less than $3500 \mathrm{~K}$, typically, means that stellar photons are unable to photodissociate or photoionise many of the common molecules produced near the stellar surface, thereby limiting their effect on chemistry. Non-smooth outflows are not only driven by stellar phenomena such as thermal pulses or surface inhomogeneities but also by the presence of (sub-)stellar companions. As shown conclusively by the ATOMIUM results ${ }^{63}$, it is highly likely that the majority of O-rich AGB stars have a stellar or planetary companion that affects the physical structure of the outflow and which may contribute to the internal UV flux that permeates the dust formation zone. While the evidence of companions objects in C-rich objects is less comprehensive, there is no reason to believe that the presence of companions will be any less 

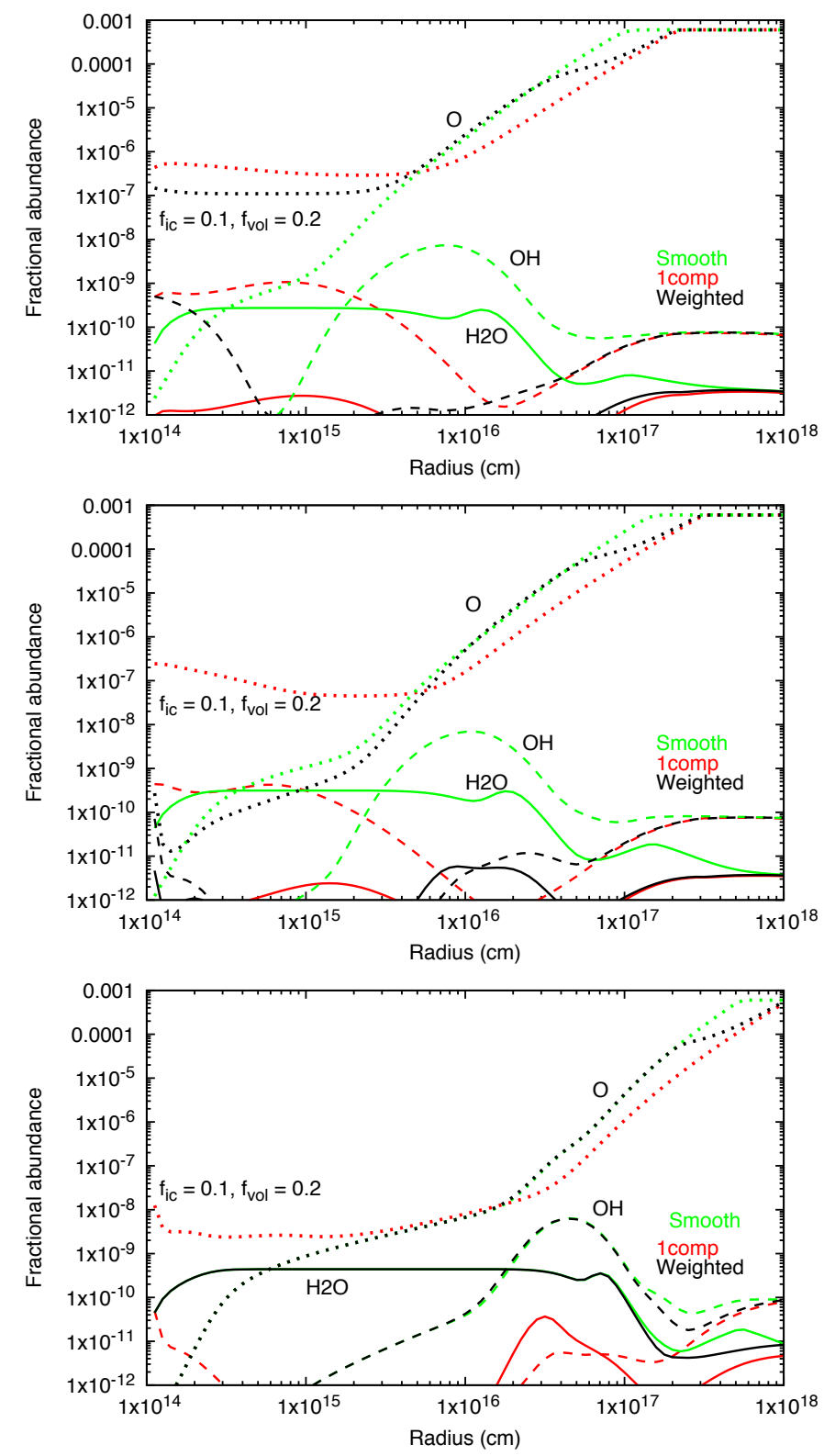

FIG. 8. Distribution of $\mathrm{H}_{2} \mathrm{O}, \mathrm{OH}$ and $\mathrm{O}$ fractional abundances for a stellar $\mathrm{BB}$ at $2330 \mathrm{~K}$ and a white dwarf companion at $6000 \mathrm{~K}$. Mass-loss rates are $10^{-6} \mathrm{M}_{\odot} \mathrm{yr}^{-1}$ (top), $2 \times 10^{-6} \mathrm{M}_{\odot} \mathrm{yr}^{-1}$ (middle), and $2 \times 10^{-5} \mathrm{M}_{\odot} \mathrm{yr}^{-1}$ (bottom). Solid lines show the $\mathrm{H}_{2} \mathrm{O}$ abundance, dashed lines that of $\mathrm{OH}$, and dotted lines $\mathrm{O}$.

likely in them than those in O-rich stars. It may be the case, however, that the nature of the density perturbations differ in both types of star due to their different dust compositions which can drive larger mass-loss rates in C-rich than in O-rich stars as carbonaceous grains are more efficient absorbers of stellar radiation than the silicates present in O-rich CSEs. 
Thus the ratio of radial momentum to orbital angular momentum can be larger in C-rich than O-rich stars, making the radially directed winds from the former harder to perturb.

Furthermore, the presence of a non-central, and non-blackbody, UV source, such as a stellar chromosphere, a (sub-)stellar companion, or an accretion disk, means that the transport of photons in a non-symmetric, clumpy medium and its effect on chemistry will be complex to model. The chemical effects of far-UV photons implies that the initial stages of dust formation may involve not only to neutral-neutral reactions, as has been commonly assumed, but also reactions between ions and neutrals. Depending on the nature of the radiation source and the wavelength dependence of the UV flux, chemistry in such regions might be much more selective than is the case in the interstellar medium. That is, only certain species may be dissociated or ionised. For example there may be no photons energetic enough to ionise carbon or sulfur atoms and, as a result, their chemistries may be more restricted than they are in interstellar clouds.

In Sect. III A, we showed that external, interstellar UV photons cannot affect the chemistry in the inner CSEs of AGB stars unless either the mass-loss rate is low, less than about $10^{-6} \mathrm{M}_{\odot} \mathrm{yr}^{-1}$, or the material is highly clumped so that the effective extinction is around two orders of magnitude less than that observed in a smooth outflow. An alternative means by which UV photons can affect the inner chemistry is to consider the role of UV photons generated by the AGB star itself (Sect. III B) or by a companion object (Sect. III C). In the former case, internal stellar photons are unable to affect the chemistry for mass-loss rates much above $10^{-6} \mathrm{M}_{\odot} \mathrm{yr}^{-1}$ due to the effects of dust extinction ${ }^{54}$. In particular, the large abundance of water detected in IRC+10216 is not reproduced in a model in which $f_{\text {vol }}=0.1$ due to the large effective extinction in the inner envelope. Indeed Fig. 5 shows that stellar photons do not alter the abundance of $\mathrm{H}_{2} \mathrm{O}$ at all for radii less than a few times $10^{16} \mathrm{~cm}$. For a much more porous envelope, for example with $f_{v o l}=0.01$, then the water abundance increases by about a factor of 3-4 at $10^{15} \mathrm{~cm}$, although still at a level about an order of magnitude less than that observed.

If the mass-loss rate is much smaller than that in $\mathrm{IRC}+10216$, for example, $10^{-6} \mathrm{M}_{\odot} \mathrm{yr}^{-1}$, then the water abundance does increase somewhat but its maximum fractional abundance is $10^{-8}$ at $10^{15} \mathrm{~cm}$, even for a highly porous outflow with $f_{\text {vol }}=0.01$, though still more than an order of magnitude less than observed (Fig. 6).

The inclusion of an internal, 'hot' source of UV photons, for example from a stellar chro- 
mosphere, a stellar companion, or an accretion disk around a companion, can change the internal abundances appreciably even for cases in which $f_{v o l}$ is relatively large, as shown in Figs. 7 and 8. The nature of the changes made depends very sensitively on the particular physical parameters chosen, including those associated with the companion - size, temperature, the wavelength dependence of the UV flux - and on those associated with the AGB star - mass-loss rate, wind velocity, clumpiness and porosity of the circumstellar envelope, for example. These sensitivities will be explored more fully in a future publication.

\section{ACKNOWLEDGMENTS}

This manuscript has been improved by the comments of the referees for which I am grateful. I would like to thank the STFC for support under grant number ST/P000312/1 as well as the organisers, in particular Dr Xiaohu Li, of the International Workshop on Astrochemistry, Xi'an, 2019, for their hospitality during my time in Xi'an.

\section{REFERENCES}

${ }^{1}$ A. L. Betz, Astrophys. J. Letts. 244, L103 (1981).

${ }^{2}$ D. M. Goldhaber and A. L. Betz, Astrophys. J. Letts. 279, L55 (1984).

${ }^{3}$ L. E. B. Johansson, C. Andersson, J. Ellder, P. Friberg, A. Hjalmarson, B. Hoglund, W. M. Irvine, H. Olofsson, and G. Rydbeck, Astron. Astrophys. 130, 227 (1984).

${ }^{4}$ D. M. Goldhaber, A. L. Betz, and J. J. Ottusch, Astrophys. J. 314, 356 (1987).

${ }^{5}$ P. J. Huggins and A. E. Glassgold, Astrophys. J. 252, 201 (1982).

${ }^{6}$ P. J. Huggins, A. E. Glassgold, and M. Morris, Astrophys. J. 279, 284 (1984).

${ }^{7}$ A. E. Glassgold, G. A. Mamon, A. Omont, and R. Lucas, Astron. Astrophys. 180, 183 (1987).

${ }^{8}$ L. A. M. Nejad, T. J. Millar, and A. Freeman, Astron. Astrophys. 134, 129 (1984).

${ }^{9}$ L. A. M. Nejad and T. J. Millar, Astron. Astrophys. 183, 279 (1987).

${ }^{10}$ I. Cherchneff and A. E. Glassgold, Astrophys. J. Letts. 419, L41 (1993).

${ }^{11}$ T. J. Millar, E. Herbst, and R. P. A. Bettens, MNRAS 316, 195 (2000).

${ }^{12}$ L. Decin, M. Agúndez, M. J. Barlow, F. Daniel, J. Cernicharo, R. Lombaert, E. De Beck, P. Royer, B. Vandenbussche, R. Wesson, E. T. Polehampton, J. A. D. L. Blommaert, W. De 
Meester, K. Exter, H. Feuchtgruber, W. K. Gear, H. L. Gomez, M. A. T. Groenewegen, M. Guélin, P. C. Hargrave, R. Huygen, P. Imhof, R. J. Ivison, C. Jean, C. Kahane, F. Kerschbaum, S. J. Leeks, T. Lim, M. Matsuura, G. Olofsson, T. Posch, S. Regibo, G. Savini, B. Sibthorpe, B. M. Swinyard, J. A. Yates, and C. Waelkens, Nature 467, 64 (2010).

${ }^{13}$ M. Agúndez, J. Cernicharo, and M. Guélin, Astrophys. J. Letts. 724, L133 (2010).

${ }^{14}$ M. Agúndez, J. Cernicharo, G. Quintana-Lacaci, L. Velilla Prieto, A. Castro-Carrizo, N. Marcelino, and M. Guélin, Astrophys. J. 814, 143 (2015).

${ }^{15}$ C. M. Sharp and G. J. Wasserburg, Geochim. Cosmochim. Acta 59, 1633 (1995).

${ }^{16}$ M. Agúndez, J. I. Martínez, P. L. de Andres, J. Cernicharo, and J. A. Martín-Gago, Astron. Astrophys. 637, A59 (2020).

${ }^{17}$ I. Cherchneff, Astron. Astrophys. 526, L11 (2011).

${ }^{18}$ I. Cherchneff, Astron. Astrophys. 545, A12 (2012).

${ }^{19}$ D. Gobrecht, I. Cherchneff, A. Sarangi, J. M. C. Plane, and S. T. Bromley, Astron. Astrophys. 585, A6 (2016).

${ }^{20}$ H. P. Gail and E. Sedlmayr, Astron. Astrophys. 206, 153 (1988).

${ }^{21}$ H. P. Gail and E. Sedlmayr, Astron. Astrophys. 347, 594 (1999).

${ }^{22}$ C. Helling and J. M. Winters, Astron. Astrophys. 366, 229 (2001).

${ }^{23}$ T. P. M. Goumans and S. T. Bromley, MNRAS 420, 3344 (2012).

${ }^{24}$ S. T. Bromley, J. C. Gómez Martín, and J. M. C. Plane, Phys. Chem. Chem. Phys. 18, 26913 (2016).

${ }^{25}$ D. Gobrecht, S. Cristallo, L. Piersanti, and S. T. Bromley, Astrophys. J. 840, 117 (2017).

${ }^{26}$ J. Boulangier, D. Gobrecht, L. Decin, A. de Koter, and J. Yates, MNRAS 489, 4890 (2019).

${ }^{27}$ A. L. Betz, R. A. McLaren, and D. L. Spears, Astrophys. J. Letts. 229, L97 (1979).

${ }^{28}$ J. J. Keady and S. T. Ridgway, Astrophys. J. 406, 199 (1993).

${ }^{29}$ V. Bujarrabal, J. Gomez-Gonzalez, and P. Planesas, Astron. Astrophys. 219, 256 (1989).

${ }^{30}$ R. Sahai and J. H. Bieging, Astron. J. 105, 595 (1993).

${ }^{31}$ D. González Delgado, H. Olofsson, F. Kerschbaum, F. L. Schöier, M. Lindqvist, and M. A. T. Groenewegen, Astron. Astrophys. 411, 123 (2003).

${ }^{32}$ L. Decin, J. Cernicharo, M. J. Barlow, P. Royer, B. Vandenbussche, R. Wesson, E. T. Polehampton, E. De Beck, M. Agúndez, J. A. D. L. Blommaert, M. Cohen, F. Daniel, 
W. De Meester, K. Exter, H. Feuchtgruber, J. P. Fonfría, W. K. Gear, J. R. Goicoechea, H. L. Gomez, M. A. T. Groenewegen, P. C. Hargrave, R. Huygen, P. Imhof, R. J. Ivison, C. Jean, F. Kerschbaum, S. J. Leeks, T. Lim, M. Matsuura, G. Olofsson, T. Posch, S. Regibo, G. Savini, B. Sibthorpe, B. M. Swinyard, B. Tercero, C. Waelkens, D. K. Witherick, and J. A. Yates, Astron. Astrophys. 518, L143 (2010).

${ }^{33}$ J. Cernicharo, L. B. F. M. Waters, L. Decin, P. Encrenaz, A. G. G. M. Tielens, M. Agúndez, E. De Beck, H. S. P. Müller, J. R. Goicoechea, M. J. Barlow, A. Benz, N. Crimier, F. Daniel, A. M. di Giorgio, M. Fich, T. Gaier, P. García-Lario, A. de Koter, T. Khouri, R. Liseau, R. Lombaert, N. Erickson, J. R. Pardo, J. C. Pearson, R. Shipman, C. Sánchez Contreras, and D. Teyssier, Astron. Astrophys. 521, L8 (2010).

${ }^{34}$ M. Agúndez, J. P. Fonfría, J. Cernicharo, C. Kahane, F. Daniel, and M. Guélin, Astron. Astrophys. 543, A48 (2012).

${ }^{35}$ J. Cernicharo, M. C. McCarthy, C. A. Gottlieb, M. Agúndez, L. Velilla Prieto, J. H. Baraban, P. B. Changala, M. Guélin, C. Kahane, M. A. Martin-Drumel, N. A. Patel, N. J. Reilly, J. F. Stanton, G. Quintana-Lacaci, S. Thorwirth, and K. H. Young, Astrophys. J. Letts. 806, L3 (2015).

${ }^{36}$ M. Van de Sande, C. Walsh, T. P. Mangan, and L. Decin, MNRAS 490, 2023 (2019).

${ }^{37}$ M. Van de Sande, C. Walsh, and T. Danilovich, MNRAS 495, 1650 (2020).

${ }^{38}$ M. Saberi, W. H. T. Vlemmings, and E. De Beck, Astron. Astrophys. 625, A81 (2019).

${ }^{39}$ X. Li, T. J. Millar, C. Walsh, A. N. Heays, and E. F. van Dishoeck, Astron. Astrophys. 568, A111 (2014).

${ }^{40}$ X. Li, T. J. Millar, A. N. Heays, C. Walsh, E. F. van Dishoeck, and I. Cherchneff, Astron. Astrophys. 588, A4 (2016).

${ }^{41}$ Astronomers measure extinction of radiation at wavelength $\lambda$ in magnitudes where $\mathrm{A}_{\lambda}$ $=1$ corresponds to a change in intensity by $100^{1 / 5}$, that is a change in extinction by 5 magnitudes corresponds to a change in intensity of 100.

${ }^{42}$ N. Mauron and P. J. Huggins, Astron. Astrophys. 452, 257 (2006).

${ }^{43}$ J. M. Brown and T. J. Millar, MNRAS 339, 1041 (2003).

${ }^{44}$ M. A. Cordiner and T. J. Millar, Astrophys. J. 697, 68 (2009).

${ }^{45}$ K. E. S. Ford, D. A. Neufeld, P. F. Goldsmith, and G. J. Melnick, Astrophys. J. 589, 430 (2003).

${ }^{46}$ D. McElroy, C. Walsh, A. J. Markwick, M. A. Cordiner, K. Smith, and T. J. Millar, 
Astron. Astrophys. 550, A36 (2013).

${ }^{47}$ Www.udfa.net.

${ }^{48}$ A. N. Heays, A. D. Bosman, and E. F. van Dishoeck, Astron. Astrophys. 602, A105 (2017).

${ }^{49}$ Www.strw.leidenuniv.nl/ ewine/photo.

${ }^{50}$ G. J. Melnick, D. A. Neufeld, K. E. S. Ford, D. J. Hollenbach, and M. L. N. Ashby, Nature 412, 160 (2001).

${ }^{51}$ M. Van de Sande, J. O. Sundqvist, T. J. Millar, D. Keller, W. Homan, A. de Koter, L. Decin, and F. De Ceuster, Astron. Astrophys. 616, A106 (2018).

${ }^{52}$ M. Van de Sande, J. O. Sundqvist, T. J. Millar, D. Keller, W. Homan, A. de Koter, L. Decin, and F. De Ceuster, Astron. Astrophys. 634, C1 (2020).

${ }^{53}$ T. J. Millar, in Journal of Physics Conference Series, Journal of Physics Conference Series, Vol. 728 (2016) p. 052001.

${ }^{54}$ M. Van de Sande and T. J. Millar, Astrophys. J. 873, 36 (2019).

${ }^{55}$ R. Montez, Jr., S. Ramstedt, J. H. Kastner, W. Vlemmings, and E. Sanchez, Astrophys. J. 841, 33 (2017).

${ }^{56}$ R. Ortiz and M. A. Guerrero, MNRAS 461, 3036 (2016).

${ }^{57}$ R. Ortiz, M. A. Guerrero, and R. D. D. Costa, MNRAS 482, 4697 (2019).

${ }^{58}$ G. Quintana-Lacaci, J. Cernicharo, M. Agúndez, L. Velilla Prieto, A. Castro-Carrizo, N. Marcelino, C. Cabezas, I. Peña, J. L. Alonso, J. Zúñiga, A. Requena, A. Bastida, Y. Kalugina, F. Lique, and M. Guélin, Astrophys. J. 818, 192 (2016).

${ }^{59}$ M. Maercker, S. Mohamed, W. H. T. Vlemmings, S. Ramstedt, M. A. T. Groenewegen, E. Humphreys, F. Kerschbaum, M. Lindqvist, H. Olofsson, C. Paladini, M. Wittkowski, I. de Gregorio-Monsalvo, and L. A. Nyman, Nature 490, 232 (2012).

${ }^{60}$ H. Kim, A. Trejo, S.-Y. Liu, R. Sahai, R. E. Taam, M. R. Morris, N. Hirano, and I. T. Hsieh, Nature Astronomy 1, 0060 (2017).

${ }^{61}$ P. Kervella, M. Montargès, S. T. Ridgway, G. Perrin, O. Chesneau, S. Lacour, A. Chiavassa, X. Haubois, and A. Gallenne, Astron. Astrophys. 564, A88 (2014).

${ }^{62}$ P. Kervella, W. Homan, A. M. S. Richards, L. Decin, I. McDonald, M. Montargès, and K. Ohnaka, Astron. Astrophys. 596, A92 (2016).

${ }^{63}$ L. Decin, M. Montargès, A. M. S. Richards, C. A. Gottlieb, W. Homan, I. McDonald, I. El Mellah, T. Danilovich, S. H. J. Wallström, A. Zijlstra, A. Baudry, J. Bolte, E. Cannon, 
E. De Beck, F. De Ceuster, A. de Koter, J. De Ridder, S. Etoka, D. Gobrecht, M. Gray, F. Herpin, M. Jeste, E. Lagadec, P. Kervella, T. Khouri, K. Menten, T. J. Millar, H. S. P. Müller, J. M. C. Plane, R. Sahai, H. Sana, M. Van de Sande, L. B. F. M. Waters, K. T. Wong, and J. Yates, Science 369, 1497 (2020).

${ }^{64}$ W. Homan, E. Cannon, M. Montargès, A. M. S. Richards, T. J. Millar, and L. Decin, Astron. Astrophys. (2020), in press.

${ }^{65}$ H. Kim and R. E. Taam, Astrophys. J. Letts. 759, L22 (2012).

${ }^{66}$ Z. Chen, A. Frank, E. G. Blackman, J. Nordhaus, and J. Carroll-Nellenback, MNRAS 468, 4465 (2017).

${ }^{67}$ I. El Mellah, J. Bolte, L. Decin, W. Homan, and R. Keppens, Astron. Astrophys. 637, A91 (2020). 